\title{
الأسمدة الزراعية: استخداماتها وأضرارها
}

\section{Chemical fertilizers in agriculture: uses and misuses}

\author{
خالا مصطفى Khaled Moustafa \\ Editor of Arabic Science Archive (arabixiv.org) محرر الأرشيف العربي العلمي \\ Email: khaled.moustafa@arabixiv.org البريد الإكتروني:
}

ملخص

ظهرت في العقود الأخيرة المُخصِّبات الزراعية الصناعية (أو ما يُعرف أيضاً بالأسمدة الكيميائية) كسمة بارزة من سمات الزراعة الحديثة لزيادة الإنتاج الزراعي، وتعوبض نقص العناصر المُغذية في التربة التي تخضع لزراعات مكثفة على مدار العام أو في أعوام متتالية. ولتحقيق الفائدة المزجوة من هذه الأسمدة، ينبغي إضافتها وفقاً لبرامج مدروسة، من حيث كمية ونوعية وتوقيت إضافة هذه الأسمدة، بما يتناسب مع طور نمو النبات وحاجته. ولكن الإسراف والاستخدام العثوائي لهذه المُخصبات كثيراً ما يسبب أضراراً ونتائج كارثية، على التربة نفسها، وعلى المحيط الحيوي والبيئي، وهذا ما يستوجب الاستخدام العقلاني والمتوازن لهذه المواد، لتجنب الأضرار التي تنجم عنها على المستوى البعيد، أو تجنبها نهائياً والاستعاضة عنها بالأسمدة العضوية ما أمكن.

\section{Abstract}

Over the past decades, the industry of chemical fertilizers became as one of the main characteristics of the modern agriculture systems to increase crop productions and to enrich the soil with mineral nutrients that could be depleted from the soil by intensive uses of lands. To attain the best results of fertilizers in agriculture, fertilizers should be added into the soil according to known qualitative and quantitative proportions that should fit the needs of growing plants and their growth stages. However, excessive uses and misuses of chemical fertilizers over years result in many environmental and health damages that need to be monitored and assessed toward reducing them and to avoid a full deterioration of arable lands.

Keywords: fertilizer, intensive agriculture, crop yields, crop rotation, crop cycle الأسمدة الزراعية، الأسمدة الكيميائية، الأسمدة العضوية، أضرار الأسمدة الكيميائية، زيادة الانتاج الزراعي، التبوير، التلوث البيئي. الدورات الزراعية، السماد الكيماوي 


\section{نبذة تاريخية عن الأسمدة الزراعية}

تُعرّف الأسمدة (أو المُخصِّبات) الزراعية بأنها مواد طبيعية أو صناعية تزوّد النبات بعناصر غذائية

ضرورية لنموه وتطوره وزيادة انتاجه. وتبعاً لمصدرها، تُصنف الأسمدة إلى صنفين رئيسيين هما: الأسمدة العضوية (طبيعية)، والأسمدة الكيميائية (صناعية). وتضم الأسمدة الطبيعية مخلفات حيوانية ونباتية، بينما تُحضّر الأسمدة الكيميائية من مواد معدنية وكيميائية في مصانع متخصصة معدة لهذا الغرض. وتصنف الأسمدة الكيميائية بدورها إلى أسدة بسيطة، تحتوي على عنصر واحد فقط (كالنتروجين مثلاً)، أو أسدة مركبة، تحتوي على أكثر من عنصر في آنٍ معاً، كالنتروجين والفوسفور (NP)، أو النتروجين والبوتاسيوم (NK)، أو النتروجين والفوسفور والبوتاسيوم معاً (NPK)، وهذه الأخيرة هي الأشهر والأكثر استخداماً في الزراعة. كما يمكن أن تكون الأسمدة الكيميائية سائلة أو صلبة أو غازيـة.

وتبعاً للكمية التي يحتاجها النبات من العناصر الغذائية المختلفة، تقسم هذه العناصر إلى عناصر غذائية رئيسية (أو كبرى)، وهي التي يحتاجها النبات بكميات كبيرة نسبياً، وعناصر ثانوية (أو صغرى)، وهي التي يحتاجها النبات بكميات صغيرة، ولكنها ضرورية أيضاً. وتضم العناصر المُغذية الرئيسية للنبات تسعة مركبات، هي: الكربون والأوكسجين والهيدروجين والنتروجين والفوسفور والبوتاسيوم والكبريت والمغنيزيوم والكالسيوم. أما العناصر الصغرى، فهي: الحديد والمنغنيز والبور والكلور النحاس والزنك والموليبيديوم. ويحصل النبات على حاجته من الكربون والأكسجين والهدروجين من الهواء والماء، لذلك لا تعتبر هذه العناصر أسمدة كيميائية، بالمعنى الحرفي أو الصناعي للكلمة، علماً أنها تثكل بمجموعها ما يقارب 90 بالمئة من وزن النبات الجاف، بينما هناك ضرورة ملحة لتوفر العناصر الأخرى في التربة، خاصة النتروجين N والفوسفور P والبوتاسيوم K، وتمثل هذه العناصر الثلاثة (NPK) العصب الرئيسي لصناعة الأسمدة الزراعية (الكيميائية) في العالم.

وقد استخدم الإنسان على مر العصور بعض الأساليب الفعّالة التي تساهم في إعادة التوازن الغذائي

إلى التربة، ووقف استنزاف العناصر الغذائية منها، كالدورات الزراعية (2) التي تعتمد على زراعة محاصيل مختلفة في نفس الحقل، والتبوير (1) الذي يعتمد على زراعة التربة موسماً أو أكثر، ثم تركها بعد ذلك موسماً

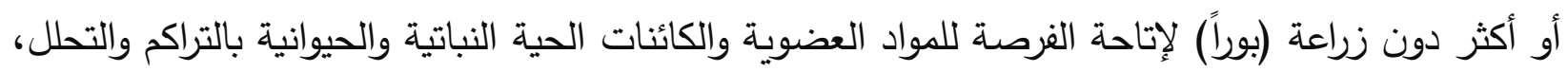
لإغناء التربة من جديد، لأن ما أخذ من التربة يعود إلى التربة، وما أخذ من الهواء يعود إلى الهواء. أما الدورات الزراعية، فتعتمد على تنويع أو مناوبة زراعة بعض الأنواع النباتية، التي تتفاوت باحتياجاتها الغذائية، والقدرة على تتبيت النتروجين الجوي من خلال ما يعرف بالتعايش مع بعض أنواع الكائنات الدقيقة التي تقوم 
بتخزين النتروجين الجوي في عقد جذرية (درنات) تتمو على جذور بعض الأنواع النباتية (خاصة البقوليات كالفاصولياء والبازلاء والفول والعدس إلخ).

ويعود استخدام الأسمدة بصورتها الطبيعية إلى تاريخ الزراعة نفسها، عندما بدأ الإنسان ممارسة الزراعة كنشاط منظم ودوري، قبل أكثر من 10 آلاف سنة، في منطقة الهلال الخصيب (بلاد الشام والرافدين)، بعد أن كان يعتمد في معظم نشاطاته على الصيد وجني الثمار والزروع، التي تتمو طبيعياً على طريق حله وترحاله، بينما بدأ استخدام الأسمدة الصناعية في القرن التاسع عشر، تزامناً مع الطفرتين الصناعية والزراعية في أوروبا (3). ثم أخذت صناعة الأسمدة طابع التصنيع التجاري على نطاق واسع، بعد الحرب العالمية الثانية. ومع ازدياد عدد سكان العالم، الذي من المتوقع أن يصل إلى ما يقارب 10 مليار نسمة بحلول عام 2050، حسب تقارير الأمم المتحدة (4)، فإن الحاجة إلى تأمين مصادر غذائية، كماً ونوعاً، تزداد باطراد. ولتلبية الطلب المتزايد على الغذاء، لا بد من زيادة الإنتاج الزراعي لمواكبة الزيادات السكانية المتوقعة، من خلال طريقتين رئيسيتين (5) (شكل 1): الأولى أفقياً، وتعني استثمار مساحات جديدة وإدخالها إلى حيز الاستثمار الزراعي، والثانية عمودياً، من خلال استخدام تقنيات حديثة تساعد على تكثيف الإنتاج في نفس المساحة المزروعة، كتربية أصناف نباتية جديدة مُهجنة عالية الانتاجية، والعمل على زيادة تحمل النبات للضغوط البيئية، كيلا تتأثر انتاجيتها كثيراً بالظروف غير المناسبة، واستخدام المخصبات الزراعية، والمكننة والآلات التي توفر كثير من التكاليف والجهد والوقت في نفس المساحة.

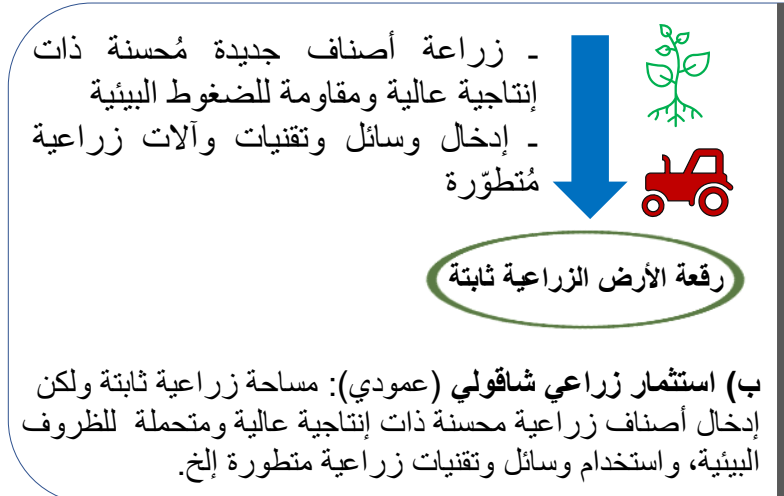

شكل (1): أنماط الاستثمار الزراعي لزيادة الإتتاج الغذائي: (أ) استثمار أفقي و (ب): استثمار شاقولي.

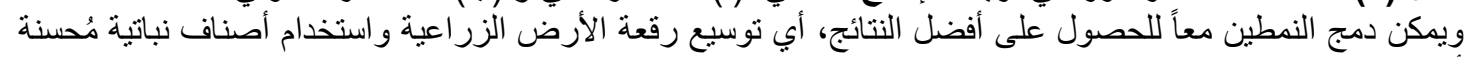
أو ذات إنتاجية عالية ووسائل وتقنيات زر اعية منطورة النيأ 
وقد أدى استخدام هذه الطرق إلى زيادات ملحوظة في الإنتاج الزراعي لمختلف المحاصيل الغذائية في

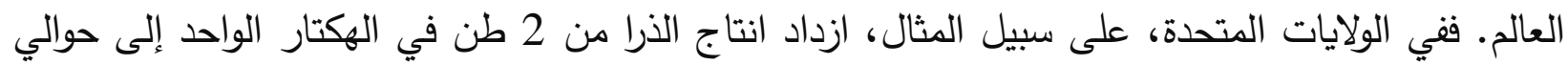

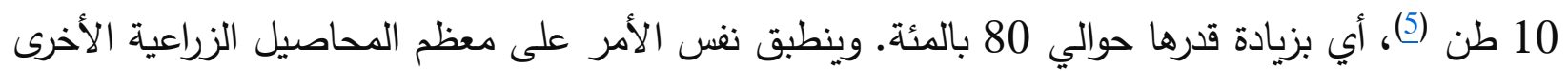

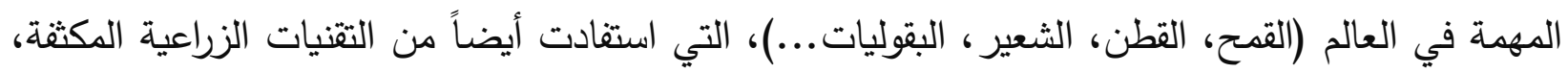
وأساليب التسميد المتنوعة، لزيادة الانتاج بنسب متفاوتة، تبعاً للبلد ودرجة التطور والعناية بالزراعة. ولكي تلبي النباتات حاجة الإنسان من الغذاء والدواء والكساء، ينبغي على الإنسان أيضاً أن يلبي حاجاتها من العناصر الغذائية والماء، عندما لا تكون هذه المصادر متوفرة بشكل طبيعي من خلال الأمطار الأنساء والأنهار والترب الخصبة. وفي ظل الزيادات السكانية المتوقعة في العقود القادمة، لن يكون الرهان حول زيادة

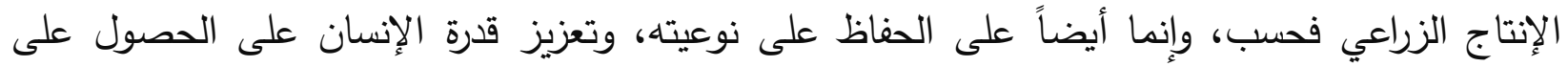

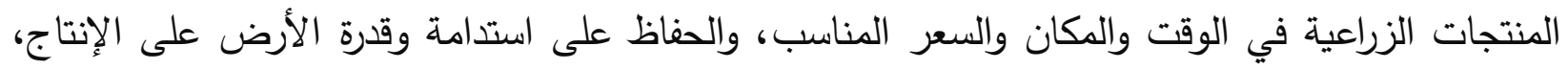

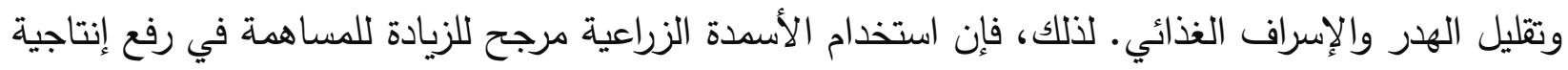

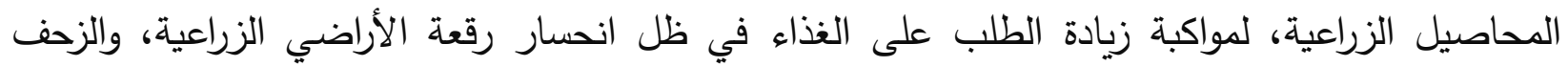
العمراني عليها، وزيادة التصحر والجفاف، وتدهور التربة الزراعية في كثير من المناطق.

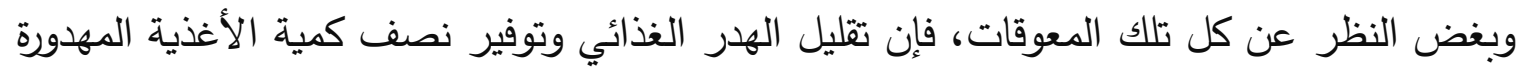
حالياً، كنفايات غذائية، يمكن أن يكفي لتغذية ما يقرب من 1,5 مليار إنسان سنوياً (6)، وهو ما يعادل

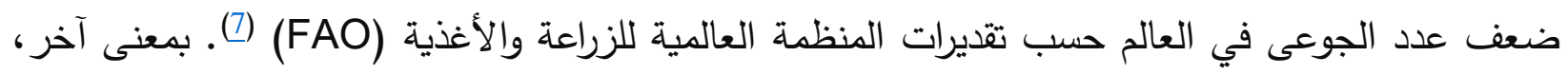

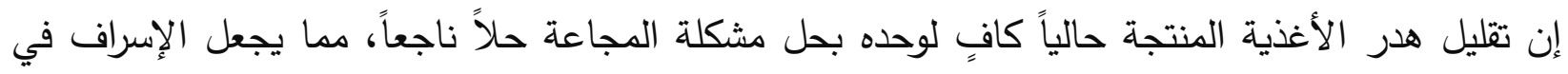

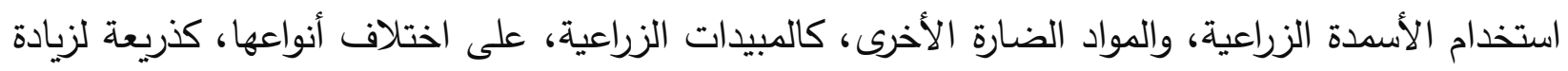

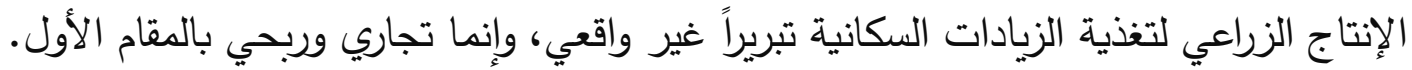
ولقد ساهم التقدم العلمي والتقني في المجالات الزراعية والتثنية في إحداث قفزة كمية ونوعية هائلة في الأساليب المتبعة في الزراعة، وتحويلها من عمل يدوي فردي لإنتاج الكفاف إلى نشاط تجاري وصناعي

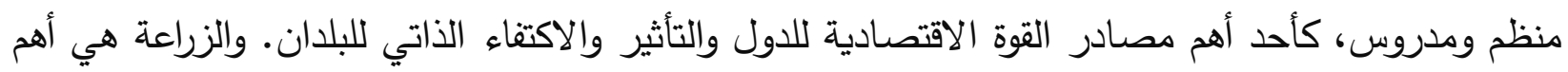
الأنثطة الاقتصادية على الإطلاق، لأنه لا يمكن للإنسان العيش دون زراعة ومنتجات نباتية وحيوانية، بينما

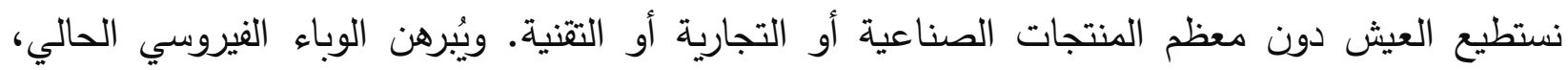
"كوفيد 19"، المنتشر على مستوى العالم، أهمية الزراعة والاكتفاء الذاتي من الأغذية والكسوة (اللباس)

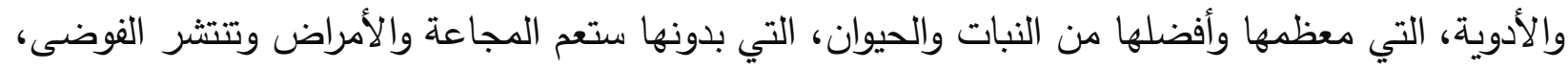
خاصة في ظل توقف الاستيراد والتجارة الدولية بسبب الحجر الصحي الدفروض على حركة البضائع والتنقل، 
لذا فالبلدان غير المنتجة زراعياً ستجد نفسها عاجزة عن تلبية احتياجات مواطنيها من الأغذية الرئيسية، أو

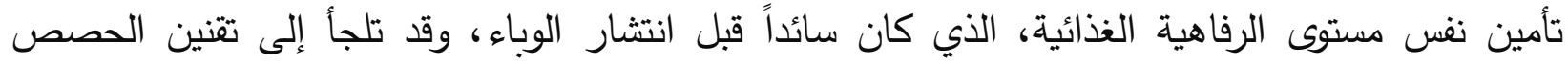
الغذائية لكل مواطن، واستيراد المنتجات الزراعية من الخارج، بأسعار مرتفعة، يمكن أن تؤدي إلى الاستدانة

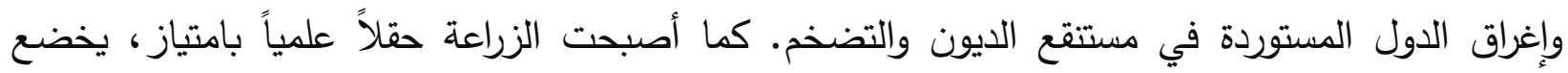
لشروط التجربة والاستقصاء والمقارنة. ولأن الزراعة هي أساس الحضارة والاستقرار، فإن التقدم الزراعي

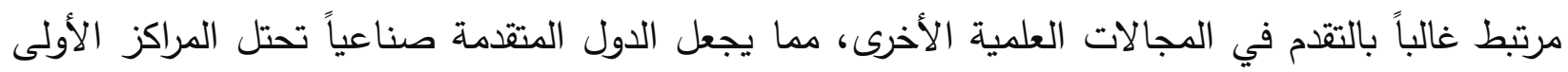
من حيث مردود الإنتاج الزراعي، والاستخدام المنظم للأسمدة الزراعية.

ميزات وعيوب الأسمدة الطبيعية والصناعية

من أهم استعمالات وميزات الأسمدة الطبيعية أنها تُحسّن تركيب التربة وبنيتها وقدرتها على امتصاص الرطوبة، والاحتفاظ بها لفترات طويلة، كما أنها نادراً ما تكون سامة أو مؤذية للنبات أو البيئة. أما أبرز عُيوبها، إن صحت تسميتها عيوباً، فهي الحاجة إلى ظروف ملائكة، من رطوبة وحرارة مناسبتين لنشاط

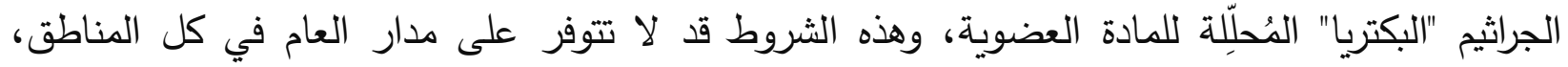
خاصة الباردة وغير المشمسة، مما يجعل فعالية هذه الأسمدة في تلك المناطق بطيئة نوعاً ما. ولكن معظم فئم البلاد العربية مشمسة ومعتدلة حرارياً، لذلك تكاد تتعدم فيها عيوب الأسمدة الطبيعية، التي تبقى ميزاتها أكبر بكثير من مساوئها في كل الظروف. أما ميزات الأسمدة الكيميائية، فهي سريعة التحلل، مما يجعل فعاليتها آنية تقريباً، وتحتوي على نسب معروفة من العناصر الغذائية المُضافة، مقارنة مع الأسمدة الطبيعية. ولكن فئن

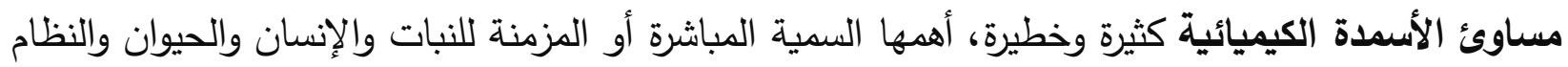

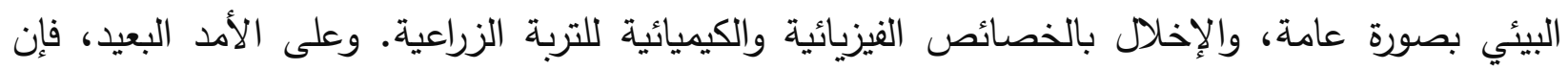
مساوئ الأسمدة الكيميائية أكبر بكثير من ميزاتها، بعكس الأسمدة الطبيعية التي تفوق ميزاتُها سيئاتها.

ويقدر حالياً استخدام الأسمدة الزراعية المكونة من العناصر الثلاثة الرئيسية (نتروجين، فوسفات، بوتاس) بحوالي 180 مليون طن في العالم، جدول (1)، معظمها في البلدان النامية وفقاً لتقارير المنظمة

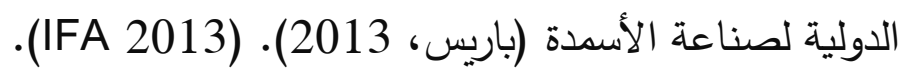

\begin{tabular}{|c|c|c|c|}
\hline 2017 & 2013 & 2012 & نوع السماد \\
\hline 115.8 & 109.4 & 107.8 & نتروجين N \\
\hline 45.0 & 40.7 & 41.3 & فوسفات (P) \\
\hline 33.2 & 29.0 & 28.9 & بوتاسيوم (K) \\
\hline 194.0 & 179.1 & 178.0 & المجموع \\
\hline
\end{tabular}

http://www.fertilizer.org المصدر: المنظمة الدولية لصناعة الأسمدة (IFA)، باريس، حزيران 2013 
ولكن، مع التتوع الكبير للترب الزراعية (رملية، ضحلة، عضوية، بركانية إلخ)، هناك بون شاسع بين تأثير الأسمدة المضافة على إنتاجية النبات في تلك الترب. ففي الترب الخصبة، جيدة التهوية، تزدهر النباتات وتعطي أفضل انتاجيتها. أما في الترب الفقيرة والضحلة، فغالباً ما تعاني النباتات وتتخفض انتاجيتها إلى الحضيض، حيث تصارع للبقاء على قيد الحياة فقط وليس للإنتاج. ولمواجهة هذه التحديات، تعتبر الأسمدة الزراعية إحدى الوسائل الآنية المتبعة لكبح نتائج تدهور التربة، ورداءة خصائصها. ولكن للأسمدة الكيميائية، كما ذكر آنفاً، سلبيات وأضرار كثيرة أيضاً، تتفاوت من حيث درجة الخطورة، وتتعلق بنوعية المنتج الزراعي، وسميتها للإنسان والحيوان والنبات، وتأثيرها السلبي على الموارد البيئية الأخرى، كالماء والهواء والدواء وهو موضوع الفقرة التالية.

\section{الأضرار البيئية والصحية للأسمدة الكيميائية}

عندما تتجاوز الكميات المضافة من الأسمدة الكيميائية نسباً معينة، وهذا ما يحدث في كثير من الأحيان من خلال إضافات متكررة وغير مدروسة، وعشوائية في كثير من البلدان، ستكون هناك تأثيرات سلبية كثيرة، مباشرة أو غير مباشرة، على النظام الحيوي خاصة والبيئي عامة. أما الانعكاسات المباشرة للأسمدة الكيميائية، فهي الأضرار المباشرة على صحة وسلامة المكونات الحية للنظام البيئي (الإنسان والحيوان والنبات). أما التأثيرات غير المباشرة، فتنعكس سلباً على مكونات النظام البيئي اللاحيوية (ماء، هواء، تربة)، حيث تؤدي إلى إحداث خلل في تركيب هذه المكونات والتوازن الطبيعي فيما بينها.

فالتسميد النتروجيني، على سبيل المثال، يعد أحد أهم التطبيقات الزراعية التي تساهم في تلويث الماء والغذاء والهواء (8). أما التسميد الفوسفاتي، على المدى الطويل، فيزيد من المخاطر البيئية والتلوث ببقايا بعض العناصر المعدنية السامة كالرصاص والزرنيخ والكادميوم (9)، ويساهم أيضاً في تعديل كثير من الخصائص الكيميائية والفيزيائية للتربة كدرجة الحموضة (pH)؛'، التي بدورها تؤثر على كمية ونوعية الكائنات الحية المفيدة. كما أن درجة حموضة التربة لها تأثثر تضادي أو تآزري بين العناصر الغذائية، حيث يزداد امتصاص بعض العناصر السمادية على حساب عناصر أخرى، عند درجات حموضـة أو قلوية معينة، فتصبح بعض العناصر غير السامة في الأصل سامة وخطيرة للنبات. كما أن درجة الحموضة ونسبة كلور الكالسيوم تؤثران على كفاءة تطهير التربة المزروعة بالرز وملوثة بالمعادن الثقيلة (10). ويكمن خطر

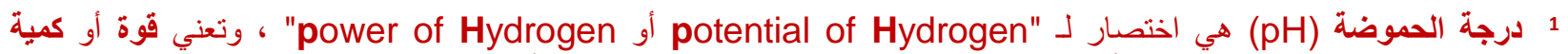

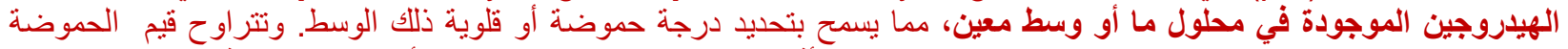

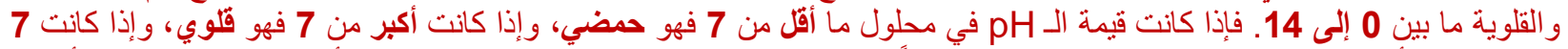

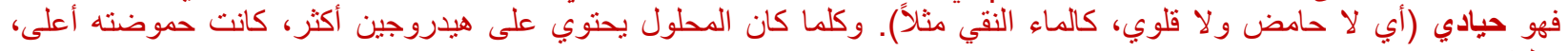


العناصر السمادية أيضاً، ومشتقاتها، في إمكانية دخولها إلى مكونات السلسلة الغذائية (نبات، حيوان، إنسان) وتركزها تراكمياً في المستويات الغذائية المتتالية، ويكون خطرها أكبر في مناطق العمران المكثف والمدن المكتظة بالسكان (11).

من ناحية أخرى، يُعدّ تسرب النترات إلى المياه الجوفية من أهم مخاطر التلوث بالسماد النتروجيني في بعض البلدان، التي تثكل فيها المياه الجوفية المصدر الرئيسي للشرب. ففي ألمانيا مثلاً، حيث 75 بالمئة من مياه الثرب هي مياه جوفية، تشير بعض التقارير إلى أن تلوث المياه الجوفية يؤدي إلى زيادة نسبة الإصابة بسرطان المعدة عند الكبار، ومتلازمة الطفل الأزرق عند الصغار (3). كما أن دورة عنصر النتروجين في الطبيعة وتسربه بين الوسط الأرضي والبحري والهوائي يؤدي إلى تشكيل عناصر أو مشتقات نتروجينية أخرى ذات سمية عالية يترسب بعضها إلى قاع المحيط، مؤثراً بذلك على البيئة البحرية ومكوناتها (12). وللتسميد المعدني طويل المدى أضرار سلبية أيضاً على تتوع بعض الفطريات الجذرية المفيدة للتربة الزراعية (13). وفي دراسة أجريت في كوريا لتقفي بعض أضرار التسميد في حقل أرز، تبين أن التسميد الآزوتي يزيد من معدل تسرب مشتقات النتروجين كالأمونيا (NH3) والأمونيوم (NH4) والنترات (NO3) والنتريت (14)(NO2) (ئ) وفي دراسات أخرى كثيرة، تبين وجود علاقة وثيقة بين مياه الشرب الملوثة بمشتقات نتروجينية،

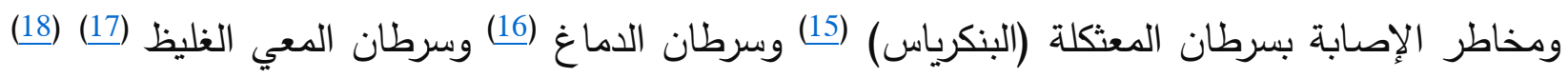

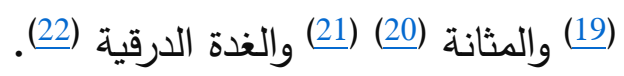

كما أن هناك تأثير غير مباشر للسماد النتروجيني على متوسط عمر بعض الطيور البحرية، التي تبين أن أعمارها تناسبت عكساً مع كميات الأسمدة المضافة في بعض المناطق الساحلية في الدانمارك، علماً أن حجم بيوضها ازداد طرداً مع نسبة السماد النتروجيني المستخدم (23). وفي دراسة حديثة أخرى، تبين أن السماد النتروجيني يؤثر ليس فقط على حجم حشرات المن التي تتغذى على نباتات مسمدة بالنتروجين، وإنما أيضاً على حجم ومعدل حياة الحشرات التي تتغذى بدورها على حشرة المن كفريسة (24)، مما يؤكد التأثير السلبي للنتروجين في السلسة الغذائية وتراكمه فيها.

وفي محاولة لمقارنة ما إذا كان تراكم العناصر السمادية الثثيلة في التربة يختلف بين حقل مكثوف وآخر مغطى، تبين أن الاستخدام المكثف طويل الأمد للأسمدة الكيميائية والعضوية في حقل خضروات مكثوف، أو ضمن بيت بلاستيكي، يؤدي إلى تراكم عناصر معدنية ثقيلة، كالخارصين (الزنك) والنحاس ومعادن أخرى، ولكن نسب التراكم في حقل مكشوف مزروع بمحصول بذري كانت أقل منها في حقل مغطى مزروع بمحاصيل خضار (25). ويمكن عزو هذا الاختلاف، بين المحاصيل البذرية والخضرية، إلى قدرة 
المحصول البذري على امتصاص وتخزين المعادن في أجزاء النبات وبذوره، مما يزيد من سمية تلك البذور عند استهلاكها.

إن استتزاف مخزون العناصر الغذائية من التربة في البلدان النامية يُعدّ أحد أهم المظاهر الخفية وراء

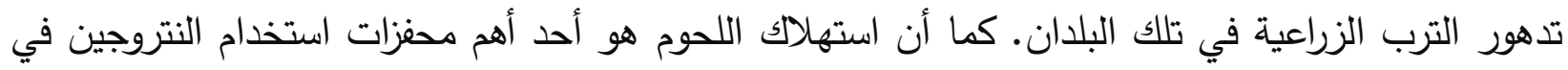
الزراعة، وبالتالي فمن المتوقع أن يزداد استخدام التسميد النتروجيني وكذلك أضراره. وتبلغ نسبة الزيادة المتوقعة في استخدام التسميد النتروجيني في الولايات المتحدة حوالي 30 بالمئة تقريباً خلال الثثلاثين سنة القادمة. وما لم يكن هناك تغيّر جذري في نمط الغذاء، والأساليب الزراعية الهادفة إلى تخفيض استخدام النتروجين، فإن زيادة استخدامه ستؤدي إلى زيادة تسرب النتروجين إلى الثواطئ المحاذية للحقول الزراعية

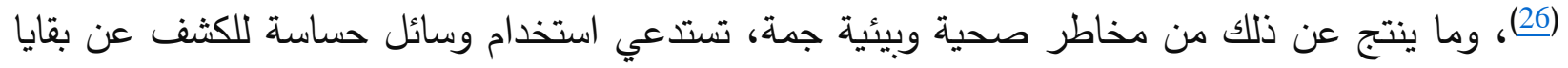
وآثار العناصر المعدنية السامة لتخفيف أضرارها قبل وقوعها، وهو موضوع الفقرة التالية.

\section{طرق الكثف عن الأسمدة الكيميائية في البيئة}

الكثف عن العناصر الضارة هو أحد أهم دعائم الإدارة الناجحة والعوامل المؤثرة في استخدام الأسمدة في النظم الزراعية التجارية، بغض النظر عن حسابات الربح والخسارة، التي غالباً ما تكون عاملاً حاسماً، وسلبياً في كثير من الأحيان، لاستخدام الأسمدة والإسراف فيها دون الآلتفات إلى الأضرار الكامنة

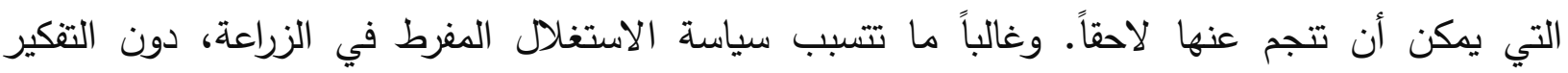
بالعواقب الآجلة أضراراً بيئية وصحية بالغة (27) تجعل من استخدام الأسمدة العضوية والكيميائية بشكل متوازن ومدروس ضرورة ملحة من أجل زراعة مستدامة وإنتاج وفير وبنوعية صحية ضمن إطار إدارة

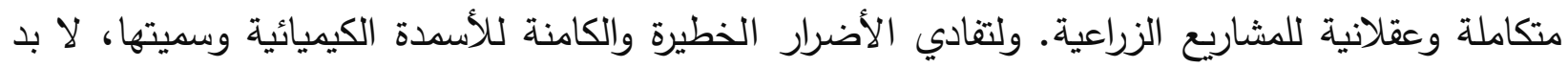
من استخدام أساليب وطرق فعالة للكشف عنها مبكراً قبل أن تتراكم بشكل غير مرئي، ويتفاقم تأثيرها وخطرها على النظام الزراعي والبيئي عموماً.

وتتباين الطرق المتبعة للكثف عن العناصر السمادية في البيئة، تبعاً لنوع العنصر السمادي المستخدم والوسط المستخدم فيه. فلتقدير كمية النترات مثلاً، يمكن استخدام طريقة إرجاع الكادميوم اللونية

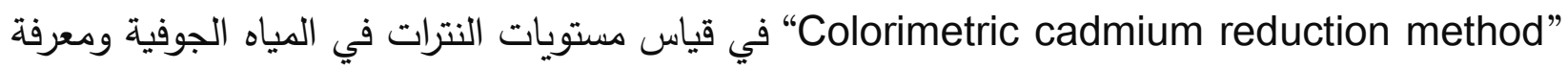
مدى تغيرها بمرور الوقت (28). كما يمكن استخدام طريقة الكثف متعددة النظائر (أي تتبّع عدة نظائر في

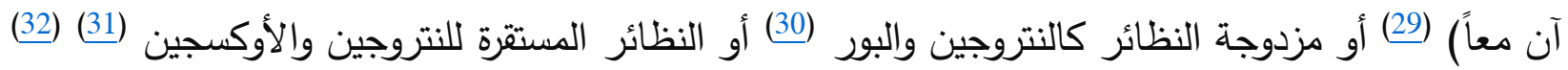
(33)، أو باستخدام أكثر من طريقة في نفس الوقت، كطريقة تتبع النظائر وكواشف كيميائية معينة، كبعض ولنض 
المستحضرات الطبية أو الغذائية (34). إلا أن طريقة تتبع النظائر تعاني من بعض العيوب، وتقل فعاليتها

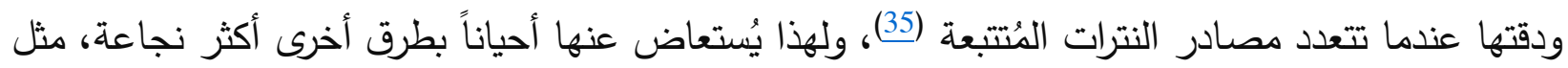

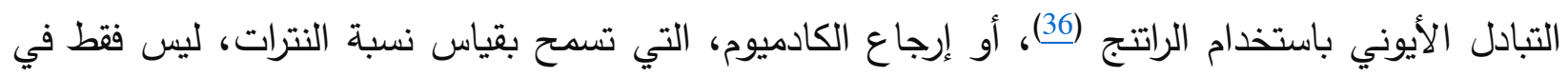

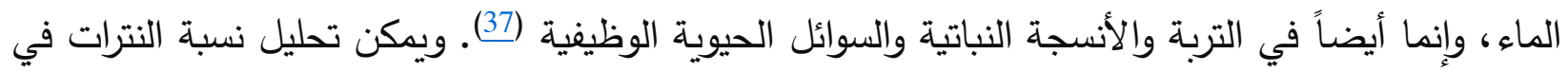
العينات الحيوية باستخدام طريقة الفصل اللوني (الكروماتوغرافيا) (38).

ومن طرق الكشف الأخرى عن العناصر السامة، طريقة تحليل طيف امتصاص الأشعة السينية، بالقرب من حافة المادة (أي قياس كمية الأشعة الممتصة في جزء سطحي، غير عميق، من المادة المُحلّة)

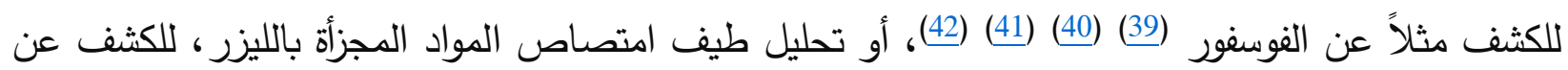
الكروم والنحاس والرصاص والزنك (43) (44).

\section{بعض الأساليب الممكنة للحد من التلوث بالأسمدة الكيميائية والعناصر السامة}

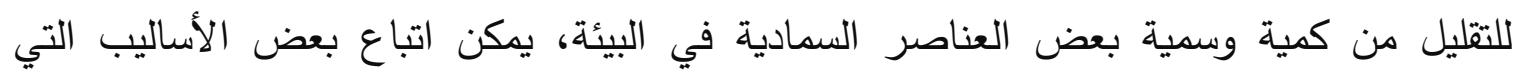

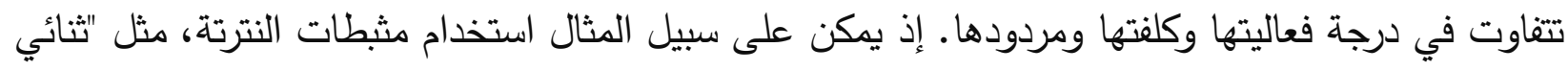

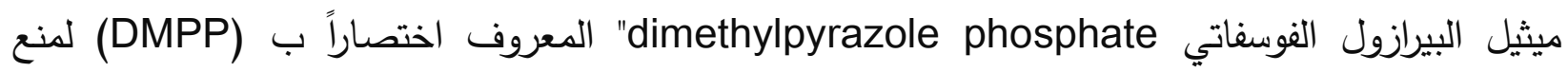
تحول النتروجين إلى نترات سامة في الأراضي التي تخضع للتسميد النتروجيني المكثف، مما يقلل من تسرب

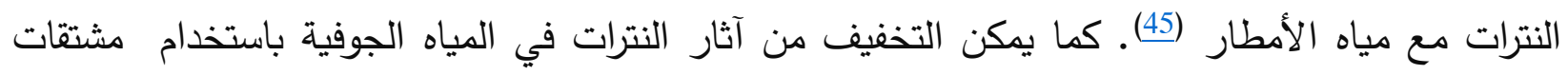
حمض الخل (الخلات) (46).

كما يمكن استخدام ثنائي أمونيوم الفوسفات (47)، أو مخلفات قثور البيض وسيقان الموز (48)، أو

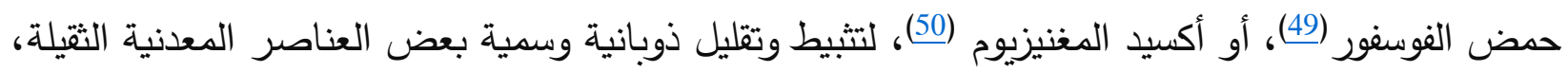
كالكادميوم والرصاص والزنك في الترب الملوثة بها، بالقرب من المصاهر المعدنية. ولكن ينبغي الحذر من

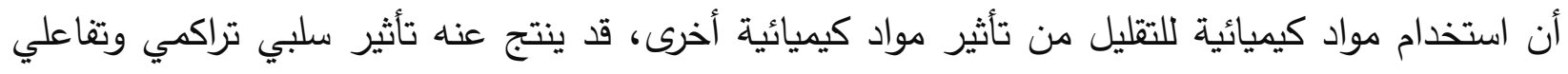

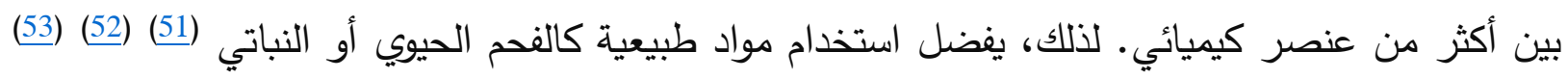
لتقليل سمية بعض العناصر المعدنية في التربة والمياه الجوفية، أو في الأوساط السائلة. ويمكن أيضاً

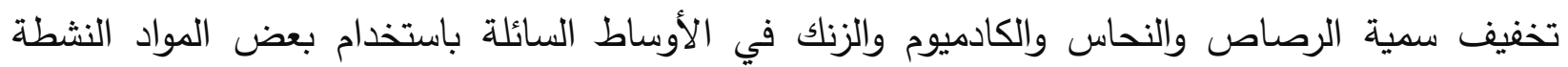

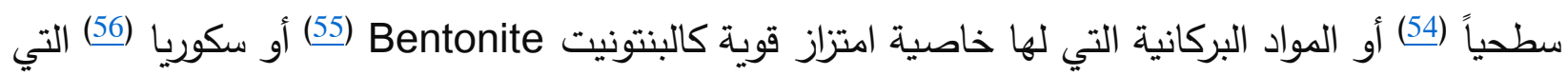
تقوم بتثبت العناصر السامة على سطحها، وبالتالي تخفف من تسربها وسميتها (سكوريا هو حجر بركاني داكن اللون إسفنجي المظهر، مسامي أو مثقب، مقاوم وعازل يتميز بالقدرة على الامتصاص والتتقية). كما 
يمكن أيضاً استخدام الفحم النباتي (أي مخلفات حرق المواد النباتية جزئياً) لإزالة الملوثات العضوية

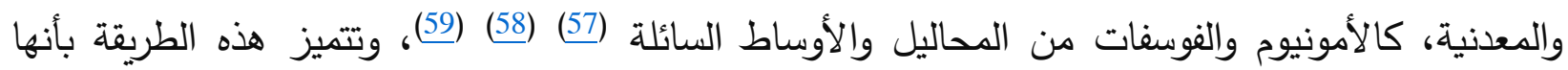
رخيصة وفعالة ومتوفرة.

وأخيراً، تبقى الطريقة الأكثر نجاعة وفعالية أماناً وأقلها كلفة وأضراراً في الحد من سمية العناصر

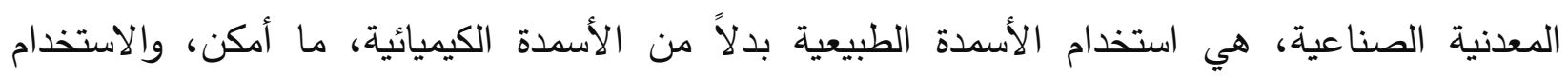

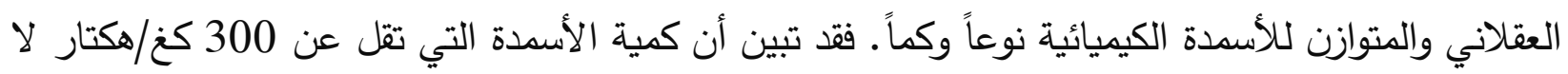

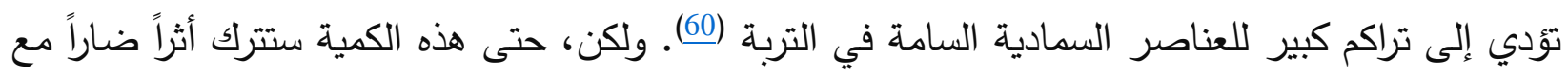
الوقت، إذا استخدمت بشكل متكرر ومتواصل لفترات طويلة، مؤدية إلى تدهور تدريجي لحالة الترب الزراعية، وقد يخرجها ذلك من حيز الاستثمار الزراعي على المدى البعيد. لذلك، فإن الإنتاج الزراعي بنوعية جيدة،

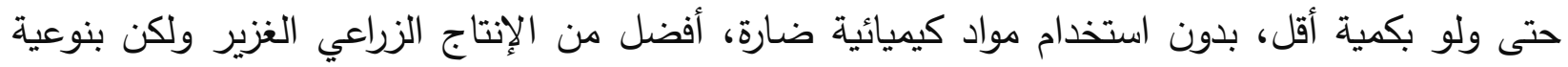

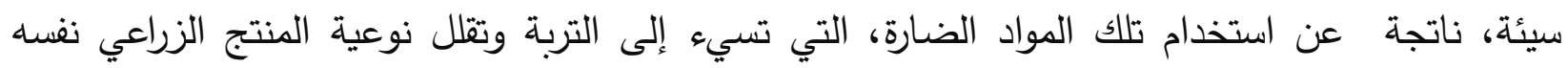
وتضر صحة الإنسان والحيوان (من طيور، وحشرات مُؤبّرة "مُلقِحِة" كالنحل، وغيرها من الحيوانات الأليفة التي تفيد البيئة والزراعة).

لذلك، فإن أفضل النظم الزراعية وأكثرها استدامة وصحة هي النظم الزراعية الطبيعية، التي لا يتم

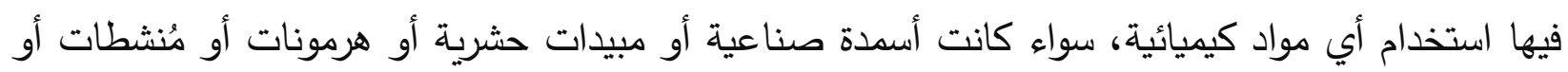
غيرها مما يبدو في ظاهره جيداً لزيادة الإنتاج وتحسين نوعيته، ولكنه ينطوي في حقيقة الأمر على أضراء

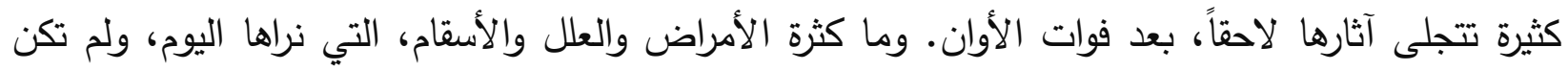
موجودة في السابق، إلا إحدى النتائج السيئة لاستخدام تلك المواد الضارة، التي كانت، ولا تزال في كثير من البلدان، تُعدُّ من مظاهر التطور والتقدم، ولكنها في جوهر الأمر، هي مواد ضارة للبيئة والصحة، من منظور

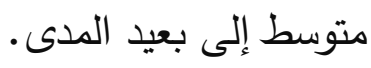

References مراجع

1. Greenland, D.J., Bringing the Green Revolution to the Shifting Cultivator. Science, 1975. 190(4217): p. 841-844. DOI: 10.1126/science.190.4217.841.

2. Bolley, H.L., Cereal Cropping: Sanitation, a New Basis for Crop Rotation, Manuring, Tillage and Seed Selection. Science, 1913. 38(973): p. 249-59. DOI: 10.1126/science.38.973.249.

3. van der Ploeg, R.R., P. Schweigert, and J. Bachmann, Use and misuse of nitrogen in agriculture: the German story. ScientificWorldJournal, 2001. 1 Suppl 2: p. 737-44. DOI: 10/1100.tsw.2001.263. 
4. World Population Prospect., World Population Prospect The 2012 Revision, in UN. 2012, United Nations: New York.

5. Edgerton, M.D., Increasing crop productivity to meet global needs for feed, food, and fuel. Plant Physiol, 2009. 149 :(1)p. 7-13. DOI: 10.1104/pp.108.130195.

6. Moustafa, K., J. Cross, and S. Gasim, Food and starvation: is Earth able to feed its growing population? Int J Food Sci Nutr, 2017: p. 1-4. DOI: 10.1080/09637486.2017.1378625.

7. FAO, I., World Food Programme "WFP", The State of Food Insecurity in the World 2015. Meeting the 2015 international hunger targets: taking stock of uneven progress. Food and Agriculture Organization Publications, Rome, 2016.

8. Lopez-Valdez, F. and F. Fernandez-Luqueno, Fertilizers components, uses in agriculture and environmental impacts. Biotechnology in Agriculture, Industry and Medicine. 2014, New York: Nova Science Publishers. 326.

9. Jiao, W., et al., Environmental risks of trace elements associated with long-term phosphate fertilizers applications: a review. Environ Pollut, 2012. 168: p. 44-53. DOI: 10.1016/j.envpol.2012.03.052.

10. Kuo, S., M.S. Lai, and C.W. Lin, Influence of solution acidity and $\mathrm{CaCl} 2$ concentration on the removal of heavy metals from metal-contaminated rice soils. Environ Pollut, 2006. 144(3): p. 918-25. DOI: 10.1016/j.envpol.2006.02.001.

11. Liu, C., et al., Recent trends in nitrogen flows with urbanization in the Shanghai megacity and the effects on the water environment. Environ Sci Pollut Res Int, 2014. DOI: 10.1007/s11356-0143825-4.

12. Fowler, D., et al., The global nitrogen cycle in the twenty-first century. Philos Trans R Soc Lond B Biol Sci, 2013. 368(1621): p. 20130164. DOI: 10.1098/rstb.2013.0164.

13. Wright, S.H., S.M. Berch, and M.L. Berbee, The effect of fertilization on the below-ground diversity and community composition of ectomycorrhizal fungi associated with western hemlock (Tsuga heterophylla). Mycorrhiza, 2009. 19(4): p. 267-76. DOI: 10.1007/s00572-008-0218-x.

14. Das, P., et al., Effect of fertilizer application on ammonia emission and concentration levels of ammonium, nitrate, and nitrite ions in a rice field. Environ Monit Assess, 2009. 154(1-4): p. 27582. DOI: 10.1007/s10661-008-0395-2.

15. Coss, A., et al., Pancreatic cancer and drinking water and dietary sources of nitrate and nitrite. Am J Epidemiol, 2004. 159(7): p. 693-701.

16. Ho, C.K., Y.H. Yang, and C.Y. Yang, Nitrates in drinking water and the risk of death from brain cancer: does hardness in drinking water matter? J Toxicol Environ Health A, 2011. 74(12): p. 74756. DOI: 10.1080/15287394.2011.539143.

17. De Roos, A.J., et al., Nitrate in public water supplies and the risk of colon and rectum cancers. Epidemiology, 2003. 14(6): p. 640-9. DOI: 10.1097/01.ede.0000091605.01334.d3.

18. McElroy, J.A., et al., Nitrogen-nitrate exposure from drinking water and colorectal cancer risk for rural women in Wisconsin, USA. J Water Health, 2008. 6(3): p. 399-409.

19. Morales-Suarez-Varela, M.M., A. Llopis-Gonzalez, and M.L. Tejerizo-Perez, Impact of nitrates in drinking water on cancer mortality in Valencia, Spain. Eur J Epidemiol, 1995. 11(1): p. 15-21.

20. Wang, W., et al., Nitrate in drinking water and bladder cancer: a meta-analysis. J Huazhong Univ Sci Technolog Med Sci, 2012. 32(6 :(p. 912-8. DOI: 10.1007/s11596-012-1057-8.

21. Ward, M.H., et al., Nitrate in public water supplies and risk of bladder cancer. Epidemiology, 2003. 14(2): p. 183-90. DOI: 10.1097/01.EDE.0000050664.28048.DF.

22. Ward, M.H., et al., Nitrate intake and the risk of thyroid cancer and thyroid disease. Epidemiology, 2010. 21(3): p. 389-95. DOI: 10.1097/EDE.0b013e3181d6201d.

23. Moller, A.P., E. Flensted-Jensen, and W. Mardal, Agriculture, fertilizers and life history of a coastal seabird. J Anim Ecol, 2007.76 :(3)p. 515-25. DOI: 10.1111/j.1365-2656.2007.01235.x. 
24. Aqueel, M.A., et al., Effect of plant nutrition on aphid size, prey consumption, and life history characteristics of green lacewing. Insect Sci, 2014. 21(1): p. 74-82. DOI: 10.1111/17447917.12019.

25. Huang, S.W. and J.Y. Jin, Status of heavy metals in agricultural soils as affected by different patterns of land use. Environ Monit Assess, 2008. 139(1-3): p. 317-27. DOI: 10.1007/s10661-0079838-4.

26. Howarth, R.W., et al., Nitrogen use in the United States from 1961-2000 and potential future trends. Ambio, 2002. 31(2): p. 88-96.

27. Moustafa, K., Exploit and ignore the consequences: A mother of planetary issues. Sci Total Environ, 2016. 557-558: p. 912-3. DOI: 10.1016/j.scitotenv.2016.03.072.

28. Ruckart, P.Z., et al., Are nitrate levels in groundwater stable over time? J Expo Sci Environ Epidemiol, 2008. 18(2): p. 129-33. DOI: 10.1038/sj.jes.7500561.

29. Widory, D., et al., Nitrate in groundwater: an isotopic multi-tracer approach. J Contam Hydrol, 2004. 72(1-4): p. 165-88. DOI: 10.1016/j.jconhyd.2003.10.010.

30. Widory, D., et al., Tracking the sources of nitrate in groundwater using coupled nitrogen and boron isotopes: a synthesis. Environ Sci Technol, 2005. 39(2): p. 539-48.

31. Singleton, M.J , et al., Tracking sources of unsaturated zone and groundwater nitrate contamination using nitrogen and oxygen stable isotopes at the Hanford site, Washington. Environ Sci Technol, 2005. 39(10): p. 3563-70.

32. Liu, C.Q., et al., Using delta15N- and delta180-values to identify nitrate sources in karst ground water, Guiyang, southwest China. Environ Sci Technol, 2006. 40(22): p. 6928-33.

33. Mattern, S., M. Sebilo, and M. Vanclooster, Identification of the nitrate contamination sources of the Brusselian sands groundwater body (Belgium) using a dual-isotope approach. Isotopes Environ Health Stud, 2011. 47(3): p. 297-315. DOI: 10.1080/10256016.2011.604127.

34. Fenech, C., et al., The potential for a suite of isotope and chemical markers to differentiate sources of nitrate contamination: a review. Water Res, 2012. 46(7): p. 2023-41. DOI: 10.1016/j.watres.2012.01.044.

35. Xue, D., et al., Present limitations and future prospects of stable isotope methods for nitrate source identification in surface- and groundwater. Water Res, 2009. 43(5): p. 1159-70. DOI: 10.1016/j.watres.2008.12.048.

36. Xing, M. and W. Liu, An improved method of ion exchange for nitrogen isotope analysis of water nitrate. Anal Chim Acta, 2011. 686(1-2): p. 107-14. DOI: 10.1016/j.aca.2010.1.1.051

37. Crutchfield, J.D. and J.H. Grove, A new cadmium reduction device for the microplate determination of nitrate in water, soil, plant tissue, and physiological fluids. J AOAC Int, 2011. 94(6): p. 1896-905.

38. Jobgen, W.S., et al., Analysis of nitrite and nitrate in biological samples using high-performance liquid chromatography. J Chromatogr B Analyt Technol Biomed Life Sci, 2007. 851(1-2): p. 71-82. DOI: 10.1016/j.jchromb.2006.07.018.

39. Liu, J., et al., Investigation of Soil Legacy Phosphorus Transformation in Long-Term Agricultural Fields Using Sequential Fractionation, $P$ K-edge XANES and Solution P NMR Spectroscopy. Environ Sci Technol, 2015. 49(1): p. 168-76. DOI: 10.1021/es504420n.

40. Sato, S., et al., Phosphorus speciation in manure and manure-amended soils using XANES spectroscopy. Environ Sci Technol, 2005. 39(19): p. 7485-91.

41. Beauchemin, S., et al., Speciation of phosphorus in phosphorus-enriched agricultural soils using $X$-ray absorption near-edge structure spectroscopy and chemical fractionation. J Environ Qual, 2003. 32(5): p. 1809-19. 
42. Giguet-Covex, C., et al., XANES spectroscopy as a tool to trace phosphorus transformation during soil genesis and mountain ecosystem development from lake sediments. Geochimica et Cosmochimica Acta, 2013. 118(0): p. 129-147. DOI: http://dx.doi.org/10.1016/i.gca.2013.04.017.

43. Dell'Aglio, M., et al., Monitoring of $\mathrm{Cr}, \mathrm{Cu}, \mathrm{Pb}, \mathrm{V}$ and $\mathrm{Zn}$ in polluted soils by laser induced breakdown spectroscopy (LIBS). J Environ Monit, 2011. 13(5): p. 1422-6. DOI: 10.1039/c0em00780c.

44. Kim, G., et al., Rapid detection of soils contaminated with heavy metals and oils by laser induced breakdown spectroscopy (LIBS). J Hazard Mater, 2013. 263 Pt 2: p. 754-60. DOI: 10.1016/j.jhazmat.2013.10.041.

45. Yu, Q., et al ,.Effects of combined application of organic and inorganic fertilizers plus nitrification inhibitor DMPP on nitrogen runoff loss in vegetable soils. Environ Sci Pollut Res Int, 2014. DOI: 10.1007/s11356-014-3366-x.

46. Calderer, M., et al., Denitrification in presence of acetate and glucose for bioremediation of nitrate-contaminated groundwater. Environ Technol, 2010. 31(7): p. 799-814. DOI: 10.1080/09593331003667741.

47. McGowen, S.L., N.T. Basta, and G.O. Brown, Use of diammonium phosphate to reduce heavy metal solubility and transport in smelter-contaminated soil. J Environ Qual, 2001. 30(2): p. 493500.

48. Ashrafi, M., et al., Immobilization of $\mathrm{Pb}, \mathrm{Cd}$, and $\mathrm{Zn}$ in a contaminated soil using eggshell and banana stem amendments: metal leachability and a sequential extraction study. Environ Sci Pollut Res Int, 2015. 22(1): p. 223-30. DOI: 10.1007/s11356-014-3299-4.

49. Cao, $\mathrm{X}$., et al., Immobilization of $\mathrm{Zn}, \mathrm{Cu}$, and $\mathrm{Pb}$ in contaminated soils using phosphate rock and phosphoric acid. J Hazard Mater, 2009. 164 :(3-2)p. 555-64. DOI: 10.1016/j.jhazmat.2008.08.034.

50. Navarro, A., E. Cardellach, and M. Corbella, Immobilization of $\mathrm{Cu}, \mathrm{Pb}$ and $\mathrm{Zn}$ in minecontaminated soils using reactive materials. J Hazard Mater, 2011. 186(2-3): p. 1576-85. DOI: 10.1016/j.jhazmat.2.010.12.039

51. Zhang, X., et al., Using biochar for remediation of soils contaminated with heavy metals and organic pollutants. Environ Sci Pollut Res Int, 2013. 20(12): p. 8472-83. DOI: 10.1007/s11356013-1659-0.

52. Liang, Y., et al., Biochar- and phosphate-induced immobilization of heavy metals in contaminated soil and water: implication on simultaneous remediation of contaminated soil and groundwater. Environ Sci Pollut Res Int, 2014. 21(6): p. 4665-74. DOI: 10.1007/s11356-013-2423-1.

53. $\mathrm{Xu}, \mathrm{X}$., et al., Removal of $\mathrm{Cu}, \mathrm{Zn}$, and $\mathrm{Cd}$ from aqueous solutions by the dairy manure-derived biochar. Environ Sci Pollut Res Int, 2013. 20(1): p. 358-68. DOI: 10.1007/s11356-012-0873-5.

54. Mao, X., et al., Use of surfactants for the remediation of contaminated soils: A review. J Hazard Mater, 2014. 285C: p. 419-435. DOI: 10.1016/j.jhazmat.2014.12.009.

55. Bereket, G., A.Z. Aro, and M.Z. ozel, Removal of Pb(II), Cd(II), Cu(II), and Zn(II) from Aqueous Solutions by Adsorption on Bentonite. J Colloid Interface Sci, 19 :(2)187 .97p. 338-43.

56. Kwon, J.S., et al., Removal of divalent heavy metals ( $\mathrm{Cd}, \mathrm{Cu}, \mathrm{Pb}$, and $\mathrm{Zn}$ ) and arsenic(III) from aqueous solutions using scoria: kinetics and equilibria of sorption. J Hazard Mater, 2010. 174(13): p. 307-13. DOI: 10.1016/j.jhazmat.2009.09.052.

57. Zeng, Z., et al., Sorption of ammonium and phosphate from aqueous solution by biochar derived from phytoremediation plants. J Zhejiang Univ Sci B, 2013. 14(12): p. 1152-61. DOI: 10.1631/jzus.B1300102.

58. Chen, B., Z. Chen, and S. Lv ,A novel magnetic biochar efficiently sorbs organic pollutants and phosphate. Bioresour Technol, 2011. 102(2): p. 716-23. DOI: 10.1016/j.biortech.2010.08.067. 
59. Mohan, D., et al., Organic and inorganic contaminants removal from water with biochar, a renewable, low cost and sustainable adsorbent--a critical review. Bioresour Technol, 2014. 160: p. 191-202. DOI: 10.1016/j.biortech.2014.01.120.

60. Murokh, V.I., [Nitrate and nitrite content of food products of plant origin grown with the use of mineral fertilizers]. Vopr Pitan, 1986(4): p. 65-7. 